\title{
Propuesta lúdica como herramienta de apoyo al proceso enseñanza - aprendizaje en el estudio del trabajo, enfocada a la estandarización de tiempos
}

\section{Playful proposal as a tool to support the teaching - learning process in the study of work, focused on the standardization of time}

\author{
Luisa Fernanda Gómez Giraldo \\ Universidad de San Buenaventura \\ luisagogi@gmail.com
}

\author{
Yohana Marcela López Rivera \\ Universidad de San Buenaventura \\ yohana.lopez@usbmed.edu.co
}

(Tipo de Artículo: Investigación Científica y Tecnológica. Recibido el 12/06/2018. Aprobado el 25/07/2018)

\begin{abstract}
Resumen. La lúdica se ha empleado en el desarrollo de la educación como una herramienta de aprendizaje significativo en el educando, este adquiriere a través de la implementación del diseño lúdico competencias para el trabajo en equipo, la planeación y ejecución de planes para alcanzar objetivos propuestos. El estudiante al combinar acción con el conocimiento fortalece su capacidad de análisis y de confianza donde es encaminado a enfrentarse con apropiación y autonomía de las posibles situaciones reales presentadas en un futuro. En el presente artículo se presenta el diseño de una herramienta lúdica que ayuda a fortalecer las competencias del estudiante de Ingeniería Industrial específicamente en la estandarización de tiempos, enmarcado al estudio del trabajo. Los estudiantes que vivieron la experiencia lúdica hacen referencia a la importancia del aprendizaje a partir de una metodología activa ya que simulando una situación real se puede aplicar conceptos a situaciones cotidianas en el mundo laboral generando nuevos conocimientos y la capacidad de análisis frente al tema estudiado construyendo por sí mismo un aprendizaje significativo del tema que se estudia.
\end{abstract}

Abstract. The playful has been used in the development of education as a significant learning tool in the learner, this acquired through the implementation of playful design skills for teamwork, planning and implementation of plans to achieve proposed objectives. The student, by combining action with knowledge, strengthens his or her capacity for analysis and confidence, where he or she is directed to face appropriation and autonomy of possible real situations presented in the future. In this article we present the design of a playful tool that helps to strengthen the competences of the Industrial Engineering student specifically in the standardization of time, framed in the study of work. The students who lived the play experience refer to the importance of learning from an active methodology, since by simulating a real situation, concepts can be applied to everyday situations in the workplace, generating new knowledge and the ability to analyze the subject studied. by itself a significant learning of the subject being studied.

Palabras clave. Herramienta metodológica; Herramienta lúdica; medición del trabajo; valoración del ritmo

Keywords. Methodological tool; Playful tool; measurement of work; Rate assessment

DOI 10.21500/20275846.3576

\section{Introducción}

En la ingeniería como en otras áreas del saber, se han implementado nuevas metodologías de aprendizaje en el ámbito magistral, donde el estudiante asume un rol activo dada la necesidad de formar personas innovadoras, creativas, críticas, capaces de analizar el sistema y de proponer soluciones, adoptando estrategias metodológicas en el aula de clase como: aprendizaje basado en problemas[1], aprendizaje significativo basado en el desarrollo de problemas [2], aprendizaje colaborativo, aprendizaje orientado en proyectos [3] y por último el aprendizaje dado por medio lúdico y de simulación [4].

Esta última metodología de enseñanza, surge como una propuesta para contribuir al fortalecimiento de las capacidades de los estudiantes al interior de las aulas de clase permitiendo experimentar por medio de un espacio dispuesto las condiciones reales de una situación específica y simulada [5].

Es por esto, que a través del equipo de investigación del programa de Ingeniería Industrial de la Universidad de San Buenaventura - Medellín, Semillero de Lúdica Aplicada a Ingeniería SLAIN, se crea una línea de estudio enfocada al diseño y desarrollo de metodologías lúdicas con miras a fortalecer el aprendizaje de los estudiantes de ingeniería.

Se desarrolla por medio del semillero SLAIN la herramienta metodológica de aprendizaje activo descrita en este artículo, la cual se convirtió en una estrategia que se torna atractiva y motivadora captando la atención del estudiante de ingeniería hacia un aprendizaje específico. 
La prueba piloto, se realiza con estudiantes de séptimo semestre de ingeniería industrial donde es implementada en la asignatura de Métodos y tiempos. Su objetivo principal fue permitir al estudiante conocer y afianzar conceptos enfocados a la medición del trabajo, además de apropiarse de la técnica valoración del ritmo de forma práctica e ilustrativa construyendo las bases de su propio conocimiento, siendo tema de vital importancia en el área industrial.

\section{Antecedentes}

Escenarios nacionales como internacionales enmarcados en el ejercicio de la ingeniería se enfrentan a un factor preocupante que se viene dando hace ya algunas décadas requiriendo otorgar un nuevo enfoque a las estrategias de enseñanza - aprendizaje de la educación en ingeniería implementando nuevas herramientas que conlleven a reducir el alto índice de deserción de los estudiantes y la baja tendencia de ingreso a carreras profesionales como lo es la ingeniería [6]. La Organización de las Naciones Unidas para la Educación, la Ciencia y la Cultura UNESCO, advierte que el poco interés de los jóvenes al elegir ingeniería como línea profesional genera un fuerte impacto para el desarrollo de un país y con ello el alcance de los retos propuestos por los Objetivos de Desarrollo del milenio ODM[6], lo que se convierte en uno de los principales desafíos internos para el sistema de educación nacional donde se deben crear estrategias para aumentar el interés de los jóvenes por la rama de la ingeniería como una opción profesional, garantizando la innovación permanente del sistema de enseñanza, lo cual ayuda a obtener una educación completa e integral. Por otro lado, se espera que para el 2020 se requiera una cifra alrededor de 20000 profesionales en ingeniería para el desarrollo de macroproyectos a los cuales le apuesta Latinoamérica, cifra preocupante ya que anualmente solo salen al mercado laboral 1000 profesionales, cifra que debe ser considerada y analizada para crear y establecer estrategias de permanecía que permitan elevarlas de forma exponencial[7]. El presidente de la Asociación Colombiana de Facultades de Ingeniería ACOFI, John William Barch, expresa su preocupación por el déficit de ingenieros existentes, los cuales, son la clave para el desarrollo económico y social del país donde solo el $28 \%$ de ellos logran culminar su carrera universitaria[8]. En Colombia, según el Sistema de Prevención y Análisis de la Deserción en las Instituciones de Educación Superior SPADIES[9], se afirma que pese al alza que han tenido las ofertas laborales para el área de ingeniería esta es la línea con mayor déficit de estudiantes competentes ya que el porcentaje de jóvenes que ingresan a las facultades de ingeniería es muy bajo además del insuficiente porcentaje de estudiantes que finalmente logran culminar su ciclo universitario.

Responder a los retos de la educación en el ingeniero del siglo XXI se hace necesario la restructuración de currículos, contenidos y métodos de aprendizaje activo donde el educando sea el actor principal de su proceso de aprendizaje[10], lo cual, sirve de apoyo a la formación de los estudiantes buscando mejorar su aprendizaje y con ello el potenciar su nivel académico. Esto es considerado importante ya que la internacionalización constante de los mercados lleva a profesionales y organizaciones a desarrollar trabajos en distintos entornos globalizados que son influenciados por aspectos políticos, económicos y sociales[11], haciendo necesario el desarrollo de herramientas que ayuden desde la perspectiva del pensamiento pedagógico moderno[12], adquirir a los estudiantes una formación significativa a través de prácticas reales facilitando la adquisición y conceptualización de nuevos conocimientos en los aprendices .

Las herramientas metodológicas son creadas para desarrollar habilidades multidisciplinarias que eviten la deserción estudiantil mostrándose como un atractivo que llama la atención por parte de los estudiantes. Estas metodologías de aprendizaje son llamadas " herramientas lúdicas"[13], las cuales, están relacionadas con modelos de aprendizaje activo siendo clave el potenciar este aprendizaje con la dirección del educador donde apoyado de la catedra magistral el estudiante tiene su primer acercamiento a conceptos básicos para construir a partir de metodologías de enseñanza - aprendizaje conocimientos y conceptos más elaborados durante su realización.

Para el desarrollo e implementación de estas herramientas enfocadas al aprendizaje activo es necesario unir la clase magistral de enseñanza tradicional con el desarrollo de la parte activa del aprendizaje, de esta necesidad surgieron dos enfoques especiales, los cuales, buscaban la renovación y mejora de los procesos educativos que se poseían. Estas metodologías de aprendizaje son la " $V$ de Gowin", donde funciona como un instrumento para aprender a aprender; y a pensar; la cual, consta de tres fases donde se parte de una pregunta de investigación que limita los alcances que se desean lograr seguido de un marco conceptual, una metodología y sus respectivas conclusiones en donde de forma concreta se debe dar a conocer el logro alcanzado dentro de su desarrollo[14]. Estas fases, ayudan a evaluar cuatro aspectos principales del aprendizaje del estudiante lo sumativo, lo formativo, lo evaluativo y por último el aspecto educativo. Por otra parte, la metodología de aprendizaje de Kolb, parte de como la experiencia influye en el aprendizaje de los estudiantes, estos deben pasar por cuatro estados durante los ciclos de aprendizaje. El primer estado es el más importante ya que se debe estar dispuesto adquirir nuevos conocimientos que sirven como base para la observación; el estudiante presenta una reflexión de lo revisado y a partir de ahí construye su conocimiento en base a la información suministrada. Durante el tercer ciclo el estudiante debe construir conceptos abstractos a partir de sus hipótesis, por último, el aprendiz debe demostrar los conocimientos adquiridos por medio de simulaciones en entornos reales, finalizados los cuatro ciclos del aprendizaje experiencial de Kolb el proceso de aprendizaje se reinicia con la primera fase experimental. 
Este estilo de aprendizaje es basado en dos dimensione principales las cuales son reflexiva y abstracta[15].

Las herramientas lúdicas se han empleado como parte del aprendizaje activo en los estudiantes de educación superior desde principios de los años 60 recreando historias que se adecuan a entornos reales para contextualizar a los estudiantes de la situación que se propone analizar. The beer game [16], forma parte de la primera implementación metodológica como apoyo a la enseñanza - aprendizaje del estudiante en el área de ingeniería, Jay Forrester crea cuatro posiciones en el diseño lúdico donde se recrea una cadena de suministro; minorista, mayorista, distribuidor y fabrica las cuales hacen parte de las estaciones que impulsan al estudiante a tomar decisiones para suavizar el efecto látigo que se presenta a lo largo de la cadena de suministro, siendo este el objetivo principal del juego ya que debido al efecto que se genera existen pérdidas a gran escala influyendo además la deficiente comunicación que puede generarse a través de ella.

El Grupo para la Enseñanza de la Investigación de Operaciones GEIO, perteneciente a la Universidad Tecnológica de Pereira, demuestra a través de estudios realizados que la enseñanza a través de herramientas lúdicas se convierten en metodologías facilitadoras para la integración de la teoría con la práctica permitiendo la construcción de conocimiento social, lo cual, no solo trabaja desde la parte cognitiva del sujeto si no que desarrolla tanto sus actitudes como su desempeño en la vida diaria y profesional [17]. Este estudio muestra, como mediante el uso de una herramienta lúdica se mejora la compresión de la planeación de requerimientos de materiales tema presentado en la asignatura planeación de la producción[18]. El diseño lúdico tiene por nombre Rouge Rever y su objetivo alcanzar era enseñar a los estudiantes a identificar los niveles que posee un documento de materiales, elemento por el cual se puede visualizar la forma de minimizar los inventarios de materia prima, subensamble y de productos finales. Los datos del estudio se generaban a partir de la producción y la demanda ya que con base a estos se pueden obtener las tasas de producción y capacidad del proceso simulado. Después de realizar la lúdica se observó que para los estudiantes que no tenían el conocimiento técnico quedaron con muchas dudas conceptuales a diferencia de los alumnos que ya poseían saberes previos respecto al tema abordado. Con la implementación de la herramienta lúdica los estudiantes lograron fijar los conceptos que se desarrollaron a lo largo de esta, asegurando que después de participar activamente de las herramientas lúdicas se logra obtener un mayor entendimiento de los procesos a desarrollar dentro de la situación simulada, estas afirmaciones son basadas respecto a las encuestas realizadas tanto al final como al inicio de la aplicación de la herramienta lúdica del grupo de investigación GEIO.

\section{Marco teórico}

El estudio de tiempos y movimientos se ha convertido en una herramienta necesaria para determinar el tiempo estándar de los procesos productivos en la industria, además de, servir como instrumento para analizar los movimientos que son ejecutados por los operarios al llevar a cabo una actividad. La implementación del estudio de tiempos y movimientos ha tenido como finalidad reducir costos empleando estrategias que eviten los movimientos innecesarios que solo generan que el tiempo de operación sea mayor. El estudio de tiempos surge durante el siglo XVIII en Francia con la elaboración de alfileres, pero es consolidado tan solo a finales del siglo XIX con las propuestas de Frederick Taylor, las cuales, fueron desarrollando el concepto de tareas en los años 80 's, donde se planteaba que la administración debería encargarse de la planeación y estandarización tanto de sus empleados como de sus procesos productivos basados en el trabajo de un operario cualificado[19].

La globalización han llevado a las organizaciones a necesitar estar en constante cambio adaptándose a las necesidades del sistema, esto implica que se incorporen desarrollos tecnológicos establecidos en herramientas aplicadas para el estudio de tiempos facilitando la labor del analista obteniendo mediciones con mayor precisión, velocidad de aplicación y resultados más confiables, compresibles y rápidos llevando a establecer un alto nivel competitivo, lo cual, han logrado a través de la selección de técnicas adecuadas para analizar sus procesos productivos, entre las técnicas más usadas en el estudio de tiempos se pueden encontrar el estudio de tiempos con cronometro donde se encuentra la técnica valoración del ritmo, tiempos básicos con cronómetros y tiempos predeterminados, además los expertos disponen de un conjunto de procedimientos que se desarrollan para el estudio de tiempos los cuales se enfocan en los registros antiguos de las actividades para establecer una tarea, estimaciones de tiempo en que dura el desarrollo de una actividad y los tiempos predeterminados que se espera se pueda realizar una actividad [20].

Dentro del estudio de tiempos nos encontramos con la técnica valoración del ritmo, la cual, no es la más considerada talvez por su nivel de dificultad pero no por ellos es de menor importancia, esta técnica se desarrolla a través de una secuencia lógica de pasos que ayudan a consolidar estándares de tiempos teniendo en cuenta el alcance de objetivos como: minimizar de los tiempos de trabajo, conservar recursos minimizando costos además de ofrecer productos mas confiables y de mayor calidad, eliminar movimientos ineficientes y acelerar los eficientes[21]

Dentro de los conceptos más representativos aplicados en la técnica de la medición del trabajo: valoración de ritmo podemos encontrar los siguientes:

- Medición del trabajo: consiste en determinar la cantidad de tiempo que invierte un trabajador 
calificado para llevar a cabo una actividad. Tiene por objetivo incrementar la eficiencia del trabajo y proporcionar estándares de tiempo que servirán de información a otros sistemas de la empresa, como el de costos de programación de la producción y supervisión [22].

- Valoración del ritmo: determina la velocidad a la que trabaja el operario por correlación con el concepto que tiene el analista de lo que es el ritmo tipo[23].

- Trabajador calificado: es aquella persona que conoce a fondo la actividad a desarrollar, además tiene la experiencia y los conocimientos de cómo hacer dicha actividad [24].

- Tiempo básico: es la cantidad de tiempo que tarda un elemento multiplicado por el valor del ritmo observado [25].

- Contenido de trabajo: es el valor del tiempo básico más la cantidad de tiempo de descanso necesario del operario para recuperarse del cansancio causado por la tarea realizada, más un tiempo suplementario por contingencia debido al trabajo [26].

- Desempeño tipo: es el rendimiento natural y sin esforzarse de un trabajador calificado en la elaboración de una actividad [27].

Es importante aclarar que la técnica antes mencionada se desarrolla a partir de varias etapas necesarias para la estandarización del ciclo de trabajo entre ellas se encuentra el obtener y registrar toda la información posible acerca de la tarea que se desea desarrollar, registrar el método con el cual se aplicara el estudio de tiempos, examinar cada parte en que se divide la tarea, medir el tiempo con un instrumento previamente revisado, determinar la velocidad de trabajo efectiva del operario permitiendo al ingeniero de métodos observar el tiempo tipo, convertir el tiempo observado de trabajo del operario en tiempo básico además determinar los suplementos que se añadirán a los tiempos básicos de cada actividad y por ultimo determinar el tiempo tipo de la operación. Las últimas dos etapas son temas de mayor relevancia en el estudio de tiempos por ende son los más discutidos por analistas e ingenieros además la valoración del ritmo de trabajo y los suplementos que se deben otorgar a los operarios para recuperarse de la fatiga son impuestos por criterios de cada organización que desee realizar el estudio de métodos por lo tanto son objeto de negociación entre trabajadores y empresarios[26].

\section{Metodología}

Para el desarrollo del caso de estudio, se siguieron una serie de pasos que conforman su metodología. Ellos se muestran en la Figura 1.

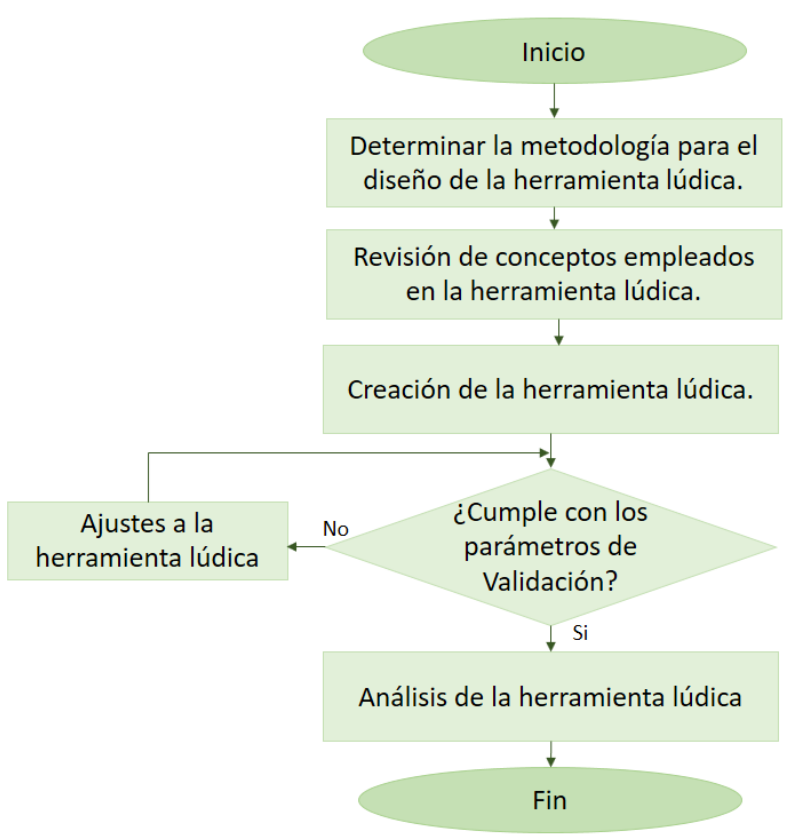

Figura 1. Diagrama de flujo de la metodología

En la primera etapa de la metodología se define la herramienta lúdica la cual se basa en el aprendizaje experiencial propuesto por Kolb y la Uve de Gowin. El primer método de enseñanza se centra en la importancia que juega la experiencia en el proceso de aprendizaje [28]. La segunda permite estructurar de forma crítica el trabajo del estudiante formando así un conocimiento significativo [29].

La herramienta lúdica sirve de apoyo en el proceso de enseñanza - aprendizaje de la medición del trabajo por lo tanto se hace necesario revisar los conceptos mas relevantes a la hora de estandarizar tiempos empleando la técnica valoración del ritmo, estos conceptos son revisados con la ayuda del docente encargado del curso Métodos y Tiempos alcanzando así el segundo paso del propósito de la metodología que se plantea.

En la siguiente etapa se estructura la lúdica, Se define el objetivo, el propósito lúdico, el producto final y sus características. Runngame, es el nombre representativo de la herramienta lúdica creada, en donde se debe ensamblar un carro con fichas tipo lego estandarizando su ciclo de trabajo, para esto se llenarán los formatos de las Tablas 4,5 y 6 . En la implementación de la herramienta lúdica serán asignados los siguientes roles: un operario quien debe realizar el ensamble del carro lego y un ingeniero de métodos quien tendrá la responsabilidad de consignar los tiempos alcanzados para cada ensamble llamado "actividad" además se debe dar la valoración según el ritmo de trabajo del operario por medio de la escala británica de valoración del ritmo tabla 1, la cual, oscilanda entre valores de 0 a 150 , si la velocidad del trabajo del operario es lenta este recibirá como valoración una calificación entre 0 y 50 de otro modo si su ritmo de trabajo es medio esta valoración estará entre 50 y 75 , si la velocidad de ritmo es constante observando al operario activo, capaz, logrando con tranquilidad el nivel de calidad y precisión 
fijado la calificación estará entre 75 y 100, si su ritmo de trabajo sobrepasa la velocidad estándar esta calificación estará en un rango de 100 a 125, por último, si el operario posee una destreza excepcional para realizar la operación sin probabilidad de durar por varios periodos realizando las actividades su calificación estará de 125 a 150. Para realizar la tarea de ensamble se deben tener claras cada una de las actividades a desarrollar tabla 2, además de conocer los materiales que se emplean para el armado el carro lego Tabla 3, cabe agregar que para el desarrollo de la herramienta lúdica se deberá establecer el trabajador cualificado quien será el operario que tenga un ritmo de trabajo estándar ya que elegir un operario lento conllevaría aumentar costos no obstante si se selecciona un operario veloz podría fatigarse y con ello afectar toda la estructura de la operación, el tiempo básico que debe obtenerse para conocer el tiempo base para cada actividad, el tiempo ciclo a estandarizar y los suplementos.

Para la validación de la herramienta de aprendizaje activo se tienen dos grupos cada uno con 17 estudiantes, estos realizan una primera encuesta para conocer los saberes previos que poseen sobre la estandarización de tiempos llevando a obtener un resultado más exacto de la eficiencia en la implementación de la herramienta lúdica permitiendo observar el impacto en el aprendizaje y la retención de información que obtienen los estudiantes. El primer grupo estudia la técnica de medición del trabajo llamada valoración del ritmo ayudados de la catedra magistral tradicional al finalizar realizan un conversatorio moderado por el docente encargado donde los 17 estudiantes exponen las dudas frente al tema visto para dar inicio a la aplicación de la herramienta lúdica Runngame. El segundo grupo solo aplica la lúdica Runngame donde a partir de la explicación del funcionamiento de la herramienta los estudiantes desarrollan su propia conceptualización frente al tema tratado. Una vez implementada la herramienta metodológica en los dos grupos, se realiza una encuesta de selección múltiple donde se validan los conceptos adquiridos por el estudiante, posterior a ello, se hace una retroalimentación por parte de los jugadores sobre la metodología y del diseño lúdico. Estos resultados obtenidos por medio de las preguntas realizadas analizan el impacto que logra obtener la implementación de la herramienta activa en los estudiantes de ingeniería.

\section{Resultados}

\subsection{Caracterización de la Lúdica}

A continuación, se describen los elementos más representativos de la herramienta lúdica creada.

- Nombre de la herramienta lúdica: Runngame.

- Logo de la herramienta lúdica:

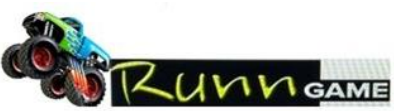

Objetivo Lúdico: Conceptuar a partir de la lúdica Runngame los conocimientos básicos de la medición del trabajo.

- Propósito lúdico: A partir del diseño lúdico generar nuevas estrategias de aprendizaje activo.

- Población objeto: Estudiantes de ingeniería industrial.

- Temática abordada: Medición de trabajo mediante la valoración del ritmo.

- Tipo de lúdica: Presencial.

- Tiempo de aplicación: 2 horas máximo.

\subsection{Descripción del desarrollo lúdico}

El prototipo final del desarrollo de la herramienta lúdica Runngame será el ensamble de un carro con fichas tipo lego como se muestra en la Figura 3, resultado de realizar las cinco actividades que se describen en la implementación de la herramienta metodológica Tabla 2. Para realizar el ensamble requerido se debe en primer instante estandarizar tanto el método con el cual se va a trabajar como los movimientos que se deben ejecutar durante el desarrollo de la actividad, además de la distribución de los insumos y materiales que se requieren en el lugar de trabajo. El puesto de trabajo estándar se observa en la Figura 4.

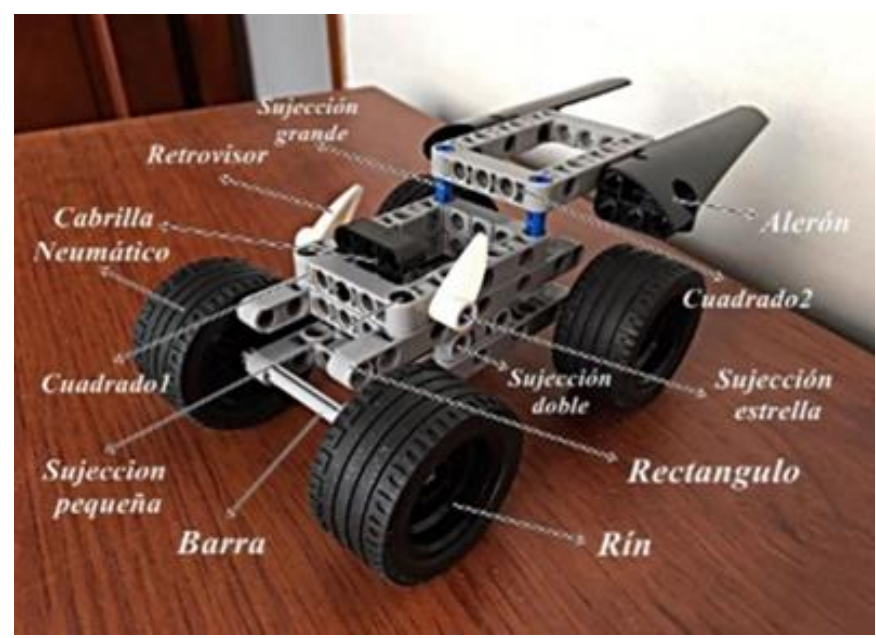

Figura 3. Patrón de ensamble

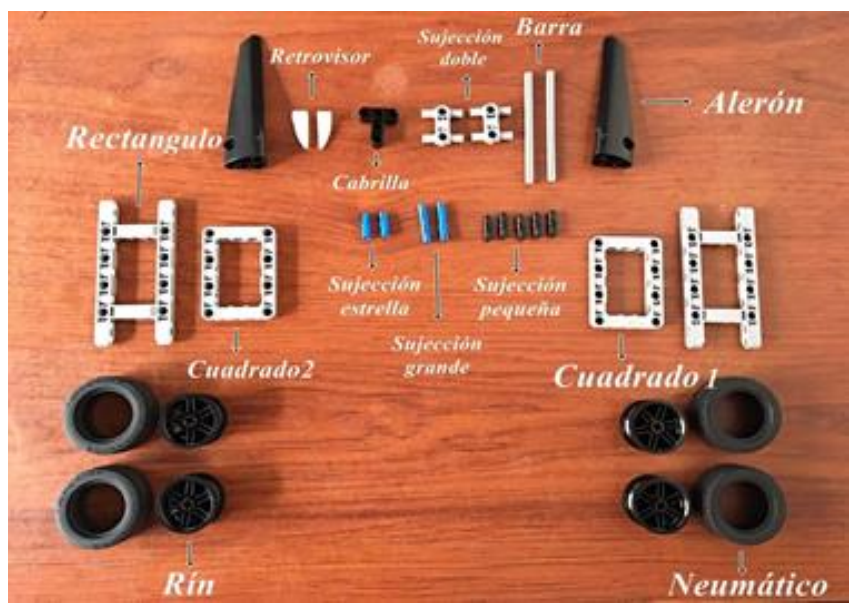

Figura 3. Puesto de trabajo estándar y Partes del carro

Figura 2. Logotipo lúdico 
Teniendo en cuenta lo anterior, el desarrollo de la herramienta metodológica es enmarcado dentro de los siguientes pasos.

Paso 1. Los jugadores deben conformar grupos de dos estudiantes por cada equipo de trabajo los cuales se repartirán los roles de operario e ingeniero de métodos, este último debe asegurarse que el operario ejecute bien el método.

Paso 2. La operación "ensamble de carro" se divide en cinco actividades: ensamble de llantas, ensamble de chasis, ensamble de carrocería 1, ensamble de carrocería 2 y ensamble de ejes. En la tabla 2, se explica el alcance de cada actividad.

Paso 3. El operario realiza de forma sistémica las actividades del paso 1, mientras el ingeniero de métodos registra los tiempos conseguidos en cada una de las actividades.

Paso 4. El estudiante que cumple el rol de ingeniero de métodos debe hacer una valoración del ritmo del trabajo del operario para cada actividad.
Paso 5. Según la valoración registrada por el analista los jugadores deben decidir quién es el operario 100.

Paso 6. A los tiempos registrados del operario $100 \mathrm{el}$ ingeniero de métodos debe otorgar suplementos por: fatiga básica $4 \%$, necesidades personales $5 \%$, contingencias $4 \%$, suplementos especiales $1 \%$.

Para fines lúdicos se propone iniciar el desarrollo de la herramienta metodológica partiendo del operario cualificado donde se debe dar la indicación de que este será el operario 100, al finalizar la implementación de la herramienta lúdica con cada uno de los ciclos a estandarizar de cada equipo se toma el menor tiempo de estos y por lo consiguiente este será el equipo ganador.

\subsection{Descripción de los insumos por actividad}

Al realizar cada actividad se deben contar con insumos tales como: neumáticos, rines, retrovisores entre otros. Los insumos requeridos para cada actividad se muestran en la Tabla 3.

Tabla 1. Escala británica de valoración del ritmo de trabajo [30]

\begin{tabular}{lll}
\hline Escala & Descripción del desempeño & $\begin{array}{l}\text { Velocidad de marcha comparable } \\
(\mathrm{Km} / \mathrm{h})\end{array}$ \\
\hline 0 & Actividad nula. & 0 \\
\hline 50 & $\begin{array}{l}\text { Muy lento, movimientos torpes, inseguros; el operario parece } \\
\text { medio dormido y sin interés por el trabajo. }\end{array}$ & 3,2 \\
\hline 75 & $\begin{array}{l}\text { Constante, resuelto, sin prisa, pero bien dirigido y vigilado: } \\
\text { parece lento, pero no pierde tiempo adrede mientras se le } \\
\text { observa. }\end{array}$ & 4,8 \\
\hline 100 (Ritmo tipo) & $\begin{array}{l}\text { Activo, capaz, logra con tranquilidad el nivel de calidad y } \\
\text { precisión establecida. }\end{array}$ & 6,4 \\
\hline 125 & $\begin{array}{l}\text { Muy rápido; actúa con gran seguridad, destreza y coordinación } \\
\text { de movimientos, muy por encima de las del obrero calificado } \\
\text { medio. }\end{array}$ \\
\hline 140 & $\begin{array}{l}\text { Excepcionalmente rápido; concentración y esfuerzo intensos } \\
\text { son probabilidad de mantenerlo por largos periodos; actuación } \\
\text { de "virtuoso", solo alcanzada por unos pocos trabajadores } \\
\text { sobresalientes. }\end{array}$ \\
\hline
\end{tabular}

Tabla 2. Alcance de cada actividad

\begin{tabular}{ll}
\hline Actividad & Alcance de cada actividad \\
\hline Ensamble de llantas & $\begin{array}{l}\text { El proceso consiste en la elaboración de } 4 \text { llantas. El ensamble inicia tomando un rin y una } \\
\text { llanta para ser ensamblados. Esta actividad es replicada } 3 \text { veces. }\end{array}$ \\
\hline Ensamble de chasis & $\begin{array}{l}\text { El proceso consiste en la elaboración del chasis. El ensamble inicia con la unión de dos } \\
\text { placas rectangulares por medio de dos sujeciones dobles en sus extremos. }\end{array}$ \\
\hline Ensamble de carrocería 1 & $\begin{array}{l}\text { El proceso consiste en la formación de la primera parte de la carrocería. El ensamble inicia } \\
\text { con la unión del cuadrado1 al chasis por medio de dos sujeciones pequeñas colocadas en } \\
\text { dos de sus extremos. Al cuadrado1, se le ensambla por medio de dos sujeciones de estrella } \\
\text { los retrovisores y por medio de una sujeción pequeña la cabrilla. }\end{array}$ \\
\hline Ensamble de carrocería 2 & $\begin{array}{l}\text { El proceso consiste en la formación de la segunda parte de la carrocería. El ensamble inicia } \\
\text { con el ensamble del cuadrado } 2 \text { al cuadrado 1 por medio de dos sujeciones largas en dos } \\
\text { de sus extremos. Al cuadrado } 2 \text { se le ensambla por medio de dos sujeciones pequeñas, dos } \\
\text { alerones. }\end{array}$ \\
\hline
\end{tabular}

Ensamble de ejes

El proceso consiste en el ensamble de los dos ejes a la carrocería y chasis. El ensamble inicia uniendo una de las cuatro llantas al carro por medio de un eje. Cada eje con dos llantas. Esta actividad es repicada una vez. 
Tabla 3. Insumos requeridos para cada actividad

\begin{tabular}{ll}
\hline Actividad & Insumos requeridos para cada actividad \\
\hline Ensamble de llantas & Cuatro neumáticos y cuatro rines. \\
\hline Ensamble de chasis & Dos rectángulos y dos sujeciones dobles. \\
\hline ensamble de carrocería 1 & Un cuadrado, dos retrovisores, una cabrilla, tres sujeciones pequeñas \\
\hline ensamble de carrocería 2 & $\begin{array}{l}\text { Un cuadrado, dos sujeciones grandes, dos sujeciones pequeñas, dos } \\
\text { alerones. }\end{array}$ \\
\hline ensamble de ejes & Cuatro llantas, dos barras, chasis, carrocería 1 y carrocería2. \\
\hline
\end{tabular}

\subsection{Diseño de formatos para registro y toma de datos}

Durante el desarrollo de la metodología de aprendizaje se debe realizar el llenado de tres formatos los cuales siguen la secuencia del proceso de estandarización del ciclo del trabajo.

En la Tabla 4, se plasman los tiempos cronometrados de cada uno de los ensambles repitiendo cuatro veces el proceso por cada actividad, luego se saca el promedio a cada una de estas respectivamente. La Tabla 5 consta del tiempo promedio de cada uno de los ensambles más la valoración y tiempo tipo. La valoración del ritmo de trabajo la da el ingeniero de métodos respecto a la observación que se obtuvo del proceso por cada actividad desarrollada además se colocara en la tabla el resultado del tiempo de la actividad o tiempo básico, una vez obtenido el tiempo de cada actividad se procede a completar la Tabla 6 , donde se colocan los suplementos, los cuales, para este desarrollo lúdico serán del $14 \%$, este porcentaje se pondrá en cada casilla de la columna suplementos y será multiplicado por el tiempo básico respectivo. Por último, el tiempo tipo será la suma del tiempo básico más el suplemento. El tiempo del ciclo que se estandariza será la sumatoria de todos los tiempos tipos establecidos por cada ensamble.

Tabla 4. Tiempos de ensamble de cada actividad con su respectivo promedio.

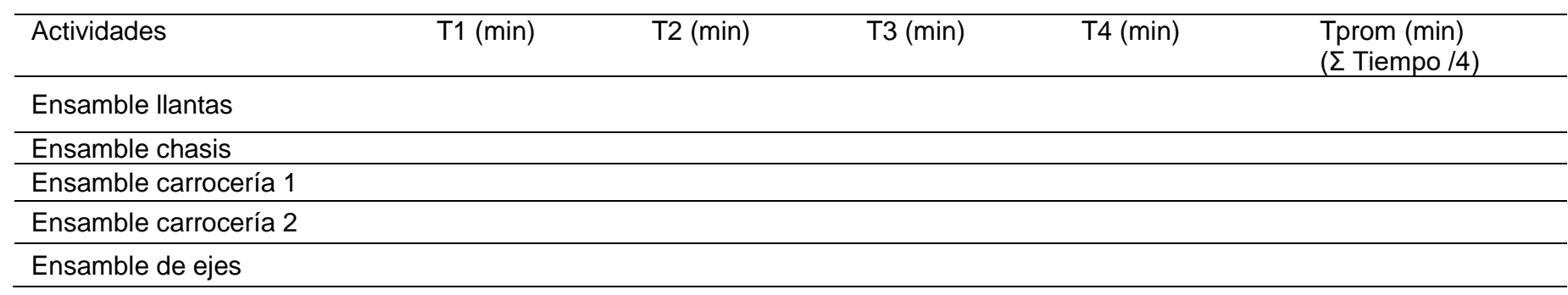

Tabla 5. Formato: tiempos básicos de ensambles.

\begin{tabular}{|c|c|c|c|}
\hline Elementos de la tarea & Tprom (min) & $\begin{array}{l}\text { Valoración (\%) } \\
\text { (según escala británica de valoración } \\
\text { del ritmo) }\end{array}$ & $\begin{array}{l}\text { Tiempo básico } \\
\text { (tpromvaloración/100) }\end{array}$ \\
\hline
\end{tabular}

Ensamble llantas

Ensamble chasis

Ensamble carrocería 1

Ensamble carrocería 2

Ensamble de ejes

Tabla 6. Formato: tiempos tipo de ensambles.

\begin{tabular}{llll}
\hline Elementos de la tarea & Tiempo básico $(\mathrm{min})$ & $\begin{array}{l}\text { Suplementos } 14 \%(\text { Tiempo básico * } \\
\text { suplementos } / 100)\end{array}$ & $\begin{array}{l}\text { Tiempo tipo }(\min ) \\
\text { (Tiempo } \\
\text { suplementos) }\end{array}$ \\
\hline Ensamble llantas & & \\
\hline Ensamble chasis & & \\
\hline Ensamble carrocería 1 & \\
\hline Ensamble carrocería 2 & \\
\hline Ensamble de ejes & & \\
\hline
\end{tabular}




\section{Análisis de resultados}

A partir de la encuesta realizada a los 34 estudiantes que participaron de la prueba piloto para comprobar la eficacia de la implementación de metodologías lúdicas como herramientas de aprendizaje activo, se obtuvieron los siguientes resultados:

El $91 \%$ de los estudiantes que participaron de la prueba piloto afirman no tener ningún tipo de conocimiento respecto a la técnica de medición del trabajo: valoración del ritmo ya que el $21 \%$ del ciento por ciento de los estudiantes que asistieron a la implementación de la herramienta metodológica pertenecientes al primer grupo ya poseían conocimientos previos al inicio de la prueba, el factor influente es que estos anteriormente ya habían cursado la asignatura de métodos y tiempos. Los resultados reflejados por las respuestas a esta pregunta se pueden observar en la Figura 5.

¿Tiene usted algun conocimiento relacionado con la tecnica de la medicion del trabajo: valoracion del ritmo?

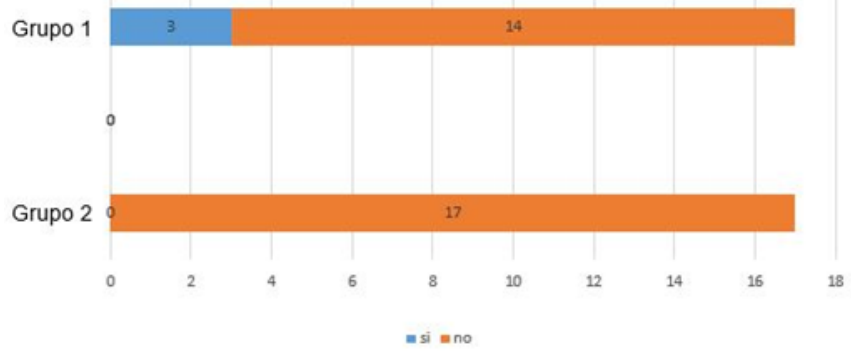

Figura 5. Respuesta del total de los estudiantes que participaron de la prueba piloto sobre el conocimiento previo de la técnica de medición del trabajo: valoración del ritmo

Por otro lado, se tiene que el primer grupo el cual recibe la catedra magistral refleja Figura 6, que al menos el $93 \%$ de los estudiantes considera que la herramienta lúdica le facilito la comprensión de la técnica de medición del trabajo: valoración del ritmo, estos realizan comentarios por medio de la encuesta de como la herramienta lúdica ayuda a entender de una forma no abstracta los conceptos abordados logrando conceptualizar el tema de una forma más visible e interesante. El estudiante que hace parte del $7 \%$ restante no logra el alcance del objetivo de la herramienta lúdica, según expresa es por aplicaciones ajenas a la implementación de la herramienta.
¿LA HERRAMIENTA LÚDICA LE FACILITO COMPRENDER LA TÉCNICA DE MEDICIÓN DEL TRABAJO: VALORACIÓN DEL RITMO?

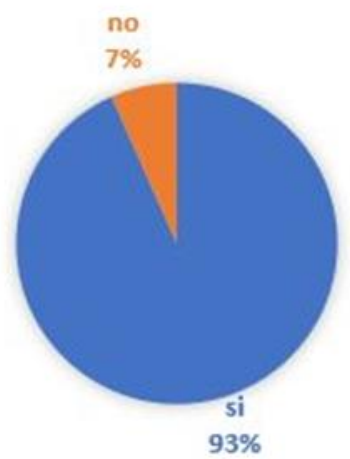

Figura 6. Porcentaje de estudiantes del primer grupo que responden a la pregunta: la herramienta lúdica le facilito comprender la técnica de medición del trabajo: valoración del ritmo

En respuesta a la pregunta de si se consideraba pertinente el uso e implementación de la herramienta de aprendizaje activo Figura 7, el total de la muestra perteneciente al segundo grupo responde que lo considera pertinente expresan de igual forma que sirve de apoyo para comprender y entender mejor los conceptos de la estandarización de tiempos. Algunos de los estudiantes agregan además que el realizar actividades de forma dinámica estas ayudan a interpretar con mayor facilidad los componentes teóricos realizando una comparación entre teoría y practica resolviendo dudas y ampliando sus conocimientos frente al tema tratado.

Como considera usted la lúdica para la enseñanza de la técnica de medición del trabajo: valoración del ritmo.

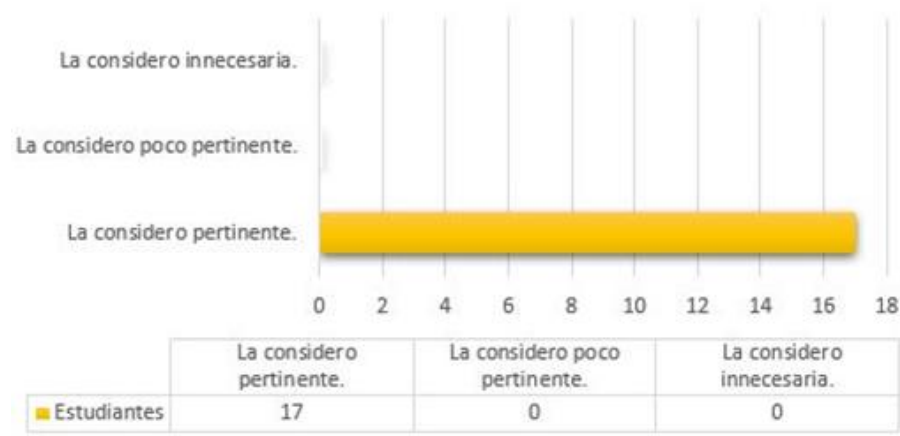

Figura 7. Número de estudiantes del segundo grupo que responden satisfactoriamente a la pregunta: como considera usted la lúdica para la enseñanza de la técnica de medición del trabajo: valoración del ritmo

Por último y como forma de validación de la lúdica se encuentra que el $100 \%$ de los estudiantes que participaron en la prueba piloto están de acuerdo en que pueden asimilar con mayor eficacia los conceptos de una forma didáctica a través de las herramientas lúdicas Figura 8, algunos estudiantes aportan que la lúdica fue 
importante porque les ayudaba a comprender conceptos que eran complejos de entender de una manera teórica.

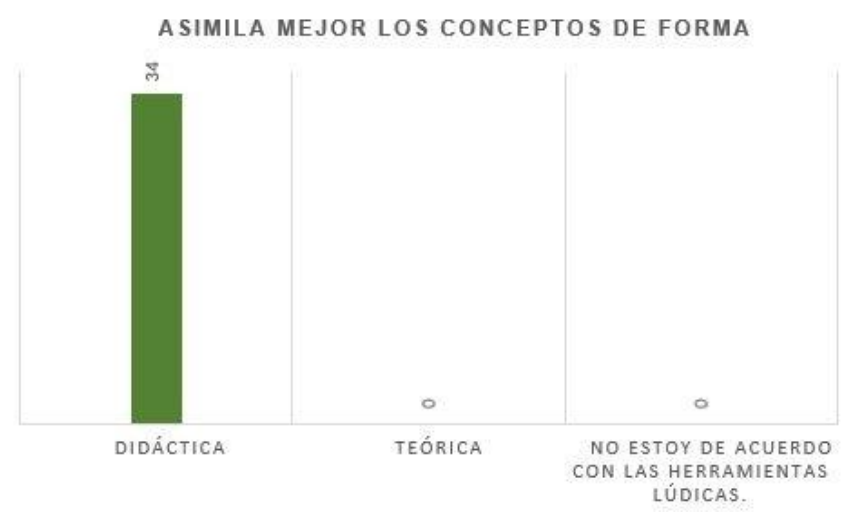

Figura 8. Frecuencia de cada una de las respuestas del grupo 1 para la pregunta: asimila mejor los conceptos de forma didáctica, teórica o no está de acuerdo con las herramientas lúdicas

Los resultados obtenidos a partir de las preguntas respondidas por los estudiantes se asemejan a los estudios realizados por [31][32], en donde se da a conocer la efectividad del desarrollo de la implementación de las herramientas lúdicas mejorando el rendimiento académico de los estudiantes comprendiendo conceptos que de otra forma serian abstractos.

Las preguntas realizadas a los grupos tenían como objetivo saber si habían podido comprender los conceptos básicos abordados en la medición del trabajo además de conocer la opinión de estos respecto a la herramienta lúdica creada y su implementación en busca de mejorar la calidad de la información que se requiere trasmitir.

\section{Conclusiones}

Existen diferencias significativas en cuanto a la adquisición del aprendizaje ya que según la evaluación realizada los estudiantes que estuvieron en la clase magistral en primera instancia presentaban fallas en la conceptualización y construcción de términos claves para la estandarización de tiempos como: cálculo de tiempo tipo, cálculo de suplementos, contenido de trabajo, cálculo de tiempo básico y factores que influyen en el ritmo de trabajo. Por otra parte, los estudiantes que participaron activamente desde el inicio de la aplicación de la herramienta lúdica se apropiaron del tema propuesto al momento de recibir la clase magistral.

Los estudiantes a través de los comentarios consignados al final de la encueta hacen referencia a la importancia del aprendizaje a partir de metodologías activas ya que simulando una situación real se pueden aplicar conceptos a situaciones cotidianas que pueden ser empleadas en el mundo laboral además de generar nuevos conocimientos y la capacidad de análisis frente a un tema estudiado donde se construye por sí mismo un aprendizaje significativo generando una visión a escala de los factores que realmente pueden influir al aplicar una técnica enseñada.

\section{Trabajos futuros}

Como trabajo futuro, se propone desarrollar una plataforma virtual para llevar acabo la lúdica de forma que facilite la implementación de otras técnicas para el desarrollo de la medición del trabajo dentro del marco del estudio de tiempos. Adema, es importante desarrollar nuevas herramientas lúdicas que garanticen el aprendizaje activo de los estudiantes sirviendo de instrumento para aprender a aprender; y a pensar.

\section{Referencia}

[1] A. Hernández Trasobares and R. Lacuesta Gilaberte, "Aplicación del aprendizaje basado en problemas (PBL) bajo un enfoque multidisciplinar: una experiencia práctica," Conoc. innovación y Emprend. camino al Futur., p. 3, 2007.

[2] I. Sánchez Soto, M. A. Moreira, and C. Caballero Sahelices, "Implementación De Una Propuesta De Aprendizaje Significativo De La Cinemática a Través De La Resolución De Problemas," Ingeniare. Rev. Chil. Ing., vol. 17, no. 1, pp. 27-41, 2009.

[3] D. R. T. C. Arias Sánchez, "Diseño de herramientas lúdicas para el apoyo de enseñanza-aprendizaje en los cursos de gestión de operaciones I y II, lean Manufacturing y administración de la producción y servicio de la UAO," Universidad autonoma de occidente, 2014.

[4] F. G. Lillo Zuñiga, "Aprendizaje Colaborativo en la Formación universitaria de pregrado," Rev. Psicol., vol. 2, no. 4, pp. 109-142, 2013.

[5] C. M. Zuluaga Ramírez, M. del P. Gómez Suta, and P. Gómez-Suta, "Metodología lúdica para la enseñanza de la programación dinámica determinista en un contexto universitario," Entramado, vol. 12, no. 1, pp. 236-249, 2016.

[6] J. M. Vélez Restrepo, P. N. Benjumea Hernández, K. J. Castro Peláez, and D. C. Ríos Echeverri, "Estrategia de Innovación en Educación en Ingeniería," pp. 1-62, 2017.

[7] Acofi, El compromiso de las facultades de ingeniería en la formación, para el desarrollo regional. 2010.

[8] L. M. Velasquez, "En Colombia se necesitan 18.000 ingenieros más," El Colomb., pp. 1-8, 2016.

[9] Universidad del los Andes, "Determinantes de la Deserción," "Informe Mens. sobre el soporte técnico y Av. del contrato para garantizar la Aliment. consolidación, validación y uso la Inf. del SPADIES, " vol. 25, no. 49, pp. 122-164, 2014.

[10] A. Camacho, "Avances en ingeniería y retos en la educación del ingeniero del siglo XXI," Third LACCEI Int. Lat. Am. Caribb. Conf. Eng. Technol., no. 11, pp. 8-10, 2005.

[11] J. M. O. TRUJILLO, "Propuesta ludica, como estrategia metodologica para la construccion de cartas de control de calidad en el aula de clase.," Universidad tecnologica de pereira, 2014.

[12] J. Cabrero, "La sociedad de la información y el conocimiento, trasformaciones tecnológicas y sus reperciosiones en la educación," Soc. la Inf. y Educ., vol. 4, pp. 62-89, 2014.

[13] L. R. Vega-González, "La educación en ingeniería en el contexto global : propuesta para la formación de ingenieros en el primer cuarto del Siglo XXI," Ing. Investig. y Tecnol., vol. XIV, no. número 2, pp. 177-190, 2013.

[14] J. Gil, F. Solano, L. M. Tobaja, and P. Monfort, "Propuesta de una herramienta didáctica basada en la $\mathrm{V}$ de Gowin para la resolución de problemas de física," Rev. Bras. Ensino Física, vol. 35, no. 2, p. 2402, 2013.

[15] R. Borracci and E. Arribalzaga, "Estilos de aprendizaje de Kolb en estudiantes de medicina," Med. (Buenos Aires), vol. 75, pp. 73-80, 2015.

[16] E. Torres, P. Uribe, and F. Valenzuela, "El juego de la cerveza," Univ. tecnológica Metrop., pp. 1-24, 2006.

[17] C. Mauricio and A. María, "GEIO automatizadas en la C elda Ingeniería y TEcnología," Entramado, vol. 10, no. 1, pp. 340-352, 2014.

[18] C. J. Mariluz Osorio Quinceno, "Utilización de la lúdica para la enseñanza del m.r.p. i," no. 32, pp. 301-306, 2006.

[19] N. L. Tejada Díaz, V. Gisbert Soler, and A. I. Pérez Molina, "Metodología De Estudio De Tiempo y Movimiento; Introducción al 
GSD Methodology of Study of Time Movement; Introduction To the Gsd," pp. 39-49, 2017.

[20] M. Rico, L. Maldonado, A. Escobedo, "Técnicas utilizadas para el estudio de tiempos: un análisis comparativo," Resvista CULCyT, no. December 2014, 2005.

[21] F. E. Myers, Estudios de tiempos y movimientos: para la manufactura gil. Pearson educación, 2011.

[22] G. Kanawaty, Introduccion al estudio del trabajo. Ginebra: Organización internacional del trabajo, 2015.

[23] T. N. Operativo, "Mejora de Métodos de Trabajo," Servicio Nacional de Adiestramiento en Trabajo Industrial, 2016.

[24] P. C. Manyoma Velásquez, "Análisis multicriterio de la valoración del ritmo en el estudio de tiempos," 4th Int. Conf. Ind. Eng. Ind. Manag., p. 9, 2010.

[25] M. Angel and G. Madurga, "Organización del trabajo," Medellin, 2015.

[26] M. Lara and E. Rodriguez, "Medicion del trabajo para un estudio de tiempos," Monterrey, 2014.
[27] R. Daniel and E. Gonzales, "Estudio Del Trabajo,” Bucaramanga, 2013.

[28] J. G. Pawelek, "El aprendizaje experiencial," Universidad de Buenos Aires, 2013.

[29] I. Sánchez, "La uve de Gowin como instrumento de aprendizaje y evaluación de habilidades de indagación en la unidad de fuerza y movimiento," scielo, vol. XXXIII, no. 2, pp. 101-125, 2012.

[30] R. García Criollo, Estudio del Trabajo. 2015.

[31] J. O. Montes de la Barrera, H. E. Hernández Riaño, J. M. López Pereira, and J. Á. Chica Urzola, "Impacto de los juegos didácticos como herramienta metodológica en el aprendizaje y la enseñanza de la ingeniería industrial," Rev. Educ. en Ing., vol. 5, no. 9, pp. 37-48, 2012

[32] L. Activities, C. M. Tool, T. O. C. P. Methods, and M. Environments, "Validación de la lúdica como herramienta metodológica complementaria en la enseñanza del método de producción tradicional y del método de producción de la teoría de restricciones ( TOC ) para el manejo de los entornos multitarea * Tool in the Teaching of," Ing. $y$ Univ., vol. 14, no. 1, pp. 97-115, 2010. 